\title{
KNOWLEDGE OF AND ATTITUDES TO EMERGENCY TACHYARRHYTHMIA TREATMENT AMONG PARAMEDICS AND NURSES
}

\author{
Maciej Sokolowski ${ }^{1}$, Magdalena Wawrzynska ${ }^{2}$ \\ 'Students' Scientific Association, Wroclaw Medical University, Wroclaw, Poland \\ ${ }^{2}$ Department of Emergency Medical Service, Laboratory of Experimental Medicine and Innovative Technology, \\ Wroclaw Medical University, Wroclaw, Poland
}

\begin{abstract}
INTRODUCTION: Supraventricular tachycardia (SVT) is a wide term used to describe tachycardia with atrial and/or ventricular rates exceeding $100 \mathrm{bpm}$ at rest. The underlying mechanism involves His bundle or tissue lying above. As the diagnosis of SVT is often made in the prehospital settings or in an emergency department, the relevant knowledge of all medical personnel, including paramedics, on the diagnosis and treatment procedures is crucial for the patient's outcome. It is essential to quickly assess the patient's clinical condition and make decisions based on both electrocardiographic records and the occurrence of adverse symptoms. Some procedures and pharmacological treatments can be implemented by the paramedic on their own, some require a physician's supervision. The aim of the study was to assess paramedics' and nurses' knowledge concerning emergency management in tachycardia.
\end{abstract}

METHODS: The study was conducted in January 2019 in south-western Poland among paramedics and nurses working in the Polish National Emergency Medical System (EMS). All participants received information on the study objectives and voluntarily took part in the questionnaire survey. The questionnaire was developed by the authors and included 22 questions referring to gender, age, work experience, type of work in EMS, number of inhabitants in the workplace area, latest training in advanced life support, adverse events in hemodynamically unstable patients, vagal manoeuvres, European Resuscitation Council 2015 guidelines for narrow and broad complex tachycardia in stable and unstable patients with regular and irregular rhythms, indications for synchronized electrical cardioversion, performing synchronized electrical cardioversion in the past, concerns about cardioversion and self-assessed level of experience in tachyarrhythmia treatment.

RESULTS: Overall, 200 paramedics and 50 nurses were asked to participate in the study. The final questionnaire return rate was $84.5 \%$. The mean age of the participants equalled as $37.90 \pm 11.44$ years; 50 (29.59\%) were female; the mean work experience was $12.07 \pm 10.39$ years. The correct treatment in a stable patient with narrow QRS tachyarrhythmia (including vassal manoeuvres and adenosine administration) was proposed by $88.17 \%$ of the participants. The correct treatment in an unstable patient with broad QRS tachyarrhythmia was proposed by $84.60 \%$ of the participants. Overall, $56.80 \%$ indicated correct treatment with a beta-blocker in a stable patient with irregular narrow complex tachyarrhythmia with a duration of over 48 hours; $87.57 \%$ knew the role of magnesium sulphate in torsade de pointes treatment. Only $34.91 \%$ had conducted electrical cardioversion in the past. According to the subjects, the main problems associated with the procedure of electrical cardioversion were: analgosedation and airway management (52.66\%), the risk for sudden cardiac arrest as a result of electrical cardioversion (53.25\%), and the risk of a stroke (38.46\%). 
The proper number of electrical cardioversions in unstable patients was proposed by $73.37 \%$ of the participants; $66.86 \%$ knew the correct dose of amiodarone when the correct electrical cardioversion sequence does not restore the sinus rhythm and the patient remains unstable. Only $43.79 \%$ knew the correct initial biphasic energy for electrical cardioversion in unstable broad complex tachyarrhythmia (120-150 J), while most responders indicated energy up to $120 \mathrm{~J}$. The mean self-assessed level of experience in tachyarrhythmia treatment was $6.00 \pm 1.76$ in a scale of $0-10$, where 0 meant lack of experience and 10 stood for experience ensuring proper performance of the procedure.

CONCLUSIONS: The knowledge of Polish paramedics and nurses concerning the emergency treatment of tachyarrhythmia is unsatisfactory in many aspects. Better undergraduate and postgraduate training on advanced life support guidelines should be recommended.

KEY WORDS: tachyarrhythmia, peri-arrest arrhythmias, emergency medicine, paramedic, nurse, medical emergency

Disaster Emerg Med J 2019; 4(1): 1-13

\section{INTRODUCTION}

Supraventricular tachycardia (SVT) is a wide term used to describe tachycardia with atrial and/or ventricular rates exceeding $100 \mathrm{bpm}$ at rest. The underlying mechanism involves His bundle or tissue lying above. As the diagnosis of SVT is often made in the prehospital settings or in an emergency department, the relevant knowledge of all medical personnel, including paramedics, on the diagnosis and treatment procedures is crucial for the patient's outcome $[1,2]$. In the event of SVT, a paramedic has to implement correct procedures, although in some cases specialist consultation is necessary. It is essential to quickly assess the patient's clinical condition and make decisions based on both electrocardiographic records and the occurrence of adverse symptoms. Some procedures and pharmacological treatments can be implemented by the paramedic on their own, some require a physician's supervision. If symptoms suggest SVT, performing the 12-lead electrocardiogram (ECG) is necessary to identify the arrhythmia. The tachycardia should first be classified according to whether there is a regular or irregular ventricular rate. If the SVT is regular, it most likely involves the atrioventricular (AV) node, being AV reentrant tachycardia (AVRT) or AV nodal reentrant tachycardia (AVNRT). An irregular ventricular rate implies atrial fibrillation (AF), which is not included in the present report. The European Resuscitation Council (ERC) guidelines suggest to evaluate the rhythm with a 12-lead ECG and to measure the QRS duration. If the QRS duration exceeds 120 ms, the tachycardia is classified as broad complex tachycardia. Otherwise, it is referred to as narrow complex tachycardia. This differentiation is essential for the choice of further treatment.

Simultaneously with ECG interpretation, the patient's hemodynamic condition must be assessed. Vagal manoeuvres and adenosine are recommended for acute treatment in hemodynamically stable patients with regular SVT. They have been shown in nonrandomized trials in an emergency department or prehospital care to effectively terminate SVT that is due to either AVNRT or AVRT, with success rates up to $90 \%$ [3]. Sinus rhythm must be promptly restored in patients with SVT who are hemodynamically unstable. Synchronized cardioversion is recommended for acute treatment in patients with hemodynamically unstable SVT. The safety and effectiveness of cardioversion in the prehospital setting was analysed in a cohort of patients with hemodynamically unstable SVT; cardioversion successfully restored sinus rhythm in all patients [4]. The guidelines advise synchronized cardioversion for any patient presenting with hypotension, acutely altered mental status, signs of shock, chest pain, or acute heart failure symptoms [5].

The ERC guidelines, defining the management of tachycardia, are directed to all medical personnel, as the assistance of an expert cardiologist is not always available.

The aim of the study was to assess the knowledge concerning emergency management in tachycardia among paramedics and nurses.

\section{MATERIAL AND METHODS}

The study protocol was approved by the institutional review board of the Polish Society of Disaster Medi- 
cine (approval No.: 12.12.2018.IRB). The study was conducted in January 2019 in south-western Poland among paramedics and nurses working in the Polish National Emergency Medical System (EMS). All participants received information on the study objectives and voluntarily took part in the questionnaire survey.

The study questionnaire was developed by the authors on the basis of their observations of paramedics training, including post-graduate training. It included 22 questions referring to gender, age, work experience, type of work in EMS, number of inhabitants in the workplace area, latest training in advanced life support, adverse events in hemodynamically unstable patients, vagal manoeuvres, ERC 2015 guidelines for narrow and broad complex tachycardia in stable and unstable patients with regular and irregular rhythms, indications for synchronized electrical cardioversion, performing synchronized electrical cardioversion in the past, concerns about cardioversion and self-assessed level of experience in tachycardia treatment.

Data were analysed with the statistical package of Statistica 13.3 (TIBCO Software Inc., Tulsa, OK, USA). Descriptive statistics were used to analyse the demographic variables. Variability was measured with the standard deviation. The normality of variable distributions was tested with the Kolmogorov-Smirnov test and the homogeneity of variance with the Fisher-Snedecor F-test and the Levene test. A conventional significance level (alpha) equal to 0.05 was applied.

\section{RESULTS}

A total of 200 paramedics and 50 nurses were asked to participate in the study. Finally, 169 questionnaires were returned (132 paramedics and 37 nurses), which means the overall return rate of $84.5 \%$. The proportion of paramedics and nurses represents the general proportions of medical personnel working in emergency departments and in medical rescue teams.

The mean age of the participants was $37.90 \pm 11.44$ years; 50 (29.59\%) were female; the mean work experience equalled $12.07 \pm 10.39$ years. Overall, $7.10 \%$ of the respondents worked in the countryside, $15.98 \%$ in a city of 20,000 inhabitants or less, $45.56 \%$ in a city of $20,000-100,000$ inhabitants, $31.36 \%$ in a city of more than 100,000 inhabitants. Among the subjects, $31.36 \%$ had participated in advanced life support training within 12 months prior to the study, $30.18 \%$ within the previous $1-3$ years, $24.85 \%$ within the previous 3-10 years, $11.24 \% 10$ or more years earlier, and $2.37 \%$ had never taken part in such a training.

The participants were surveyed for the knowledge of adverse signs in the tachycardia treatment protocol; $81.07 \%$ knew the adverse signs. The correct treatment in a stable patient with narrow QRS tachycardia (including vassal manoeuvres and adenosine administration) was proposed by $88.17 \%$ of the participants. The correct treatment in an unstable patient with broad QRS tachycardia was proposed by $84.60 \%$ of the participants. Overall, $56.80 \%$ indicated correct treatment with a beta-blocker in a stable patient with irregular narrow complex tachycardia with a duration of over 48 hours; $87.57 \%$ knew the role of magnesium sulphate in torsade de pointes treatment.

Only $34.91 \%$ of the study subjects had conducted electrical cardioversion in the past.

According to the subjects, the main problems associated with the procedure of electrical cardioversion were: analgosedation and airway management $(52.66 \%)$, the risk for sudden cardiac arrest as a result of electrical cardioversion (53.25\%), and the risk of a stroke (38.46\%).

The proper number of electrical cardioversions in unstable patients was proposed by $73.37 \%$ of the participants; $66.86 \%$ knew the correct dose of amiodarone when the correct electrical cardioversion sequence does not restore the sinus rhythm and the patient remains unstable.

Only $43.79 \%$ of the participants knew the correct initial biphasic energy for electrical cardioversion in unstable broad complex tachycardia (120-150 J), while most responders indicated energy up to $120 \mathrm{~J}$.

In the opinion of $20.70 \%$ of the study participants, the overall rate of ambulance calls for cases of tachyarrhythmia was below 1 per week, while $1-3$ calls per week were indicated by $26.63 \%$, 4-10 calls per week by $49.71 \%$, and above 10 emergency calls per week by $2.96 \%$.

The mean self-assessed level of experience in tachycardia treatment was $6.00 \pm 1.76$ in a scale of $0-10$, where 0 meant lack of experience and 10 stood for experience ensuring proper performance of the procedure.

\section{DISCUSSION}

The current ERC guidelines [6] recommend the treatment in narrow and broad complex tachycardia in 
stable and unstable patients. SVT is a common cause of acute hospital presentations [7].

Park et al. [8] showed that a considerable part of EMS personnel does not have appropriate knowledge on treating critical illness, including tachycardia in sepsis care.

Smith et al. [9] analysed the effectiveness of current prehospital SVT management by ambulance paramedics in Australia. They revealed that paramedics correctly identified SVT in $96.7 \%$ of cases. Valsalva manoeuvre was undertaken in $24.0 \%$ patients and reverted the SVT in $27.7 \%$ of attempts. Finally, $49.7 \%$ of patients remained in SVT on arrival at the hospital. Many paramedics and nurses are concerned about the emergency treatment of tachycardia. In our study, the mean self-assessed level of experience in tachycardia treatment was only 6.00 in a $0-10$ scale.

From a practical point of view, the procedure of electric cardioversion is particularly important. The authors' observations show that many paramedics and nurses are concerned about the necessity of patient sedation and the risk of complications associated with electrical cardioversion if it is not possible for a physician to supervise the procedure directly. In our study, only $43.79 \%$ of participants knew the correct initial biphasic energy for electrical cardioversion. Approximately half of the responders were concerned about analgosedation and airway management and the risk of sudden cardiac arrest as a result of electrical cardioversion.

Honarbakhsh et al. [7] compared paramedicwith the hospital-delivered treatment of acute SVT with adenosine. They revealed that patients with SVT could be effectively and safely treated by paramedics with the use of adenosine. In our study, most participants knew the indication and dosage of adenosine.

Clementy et al. [10] studied ECG monitoring in outpatient cardiac unit patients presenting with palpitations and revealed that the cause of palpitations was found in $81 \%$ of cases $(77 \%$ of them were atrial arrhythmias).

Goebel et al. [11] observed that the inappropriate use of adenosine occurred in $20 \%$ of cases and concluded that the errors in rhythm interpretation were lower among paramedics participating in targeted education programs on tachydysrhythmias.

Vagal manoeuvres are frequently applied as the first-line management tool in SVT in both emergency medicine and prehospital emergency-care settings
[12]. Smith and Boyle [13] analysed the knowledge of Melbourne's paramedics concerning the vagal manoeuvres and concluded that they would benefit from the introduction of an evidence-based model of vagal manoeuvres performance.

In our study, only $34.91 \%$ of the participants had conducted electrical cardioversion in the past. Even in the United States, some of the critical procedures including electrical cardioversion may be infrequently performed [14]. We noticed that the participants' knowledge on the energy level for cardioversion and a proper number of electrical cardioversions in unstable patients was incorrect in 1/2-1/3 of cases.

Minhas et al. [15] analysed cases of paroxysmal SVT treated in a prehospital setting. They revealed that EMS agencies typically required patients treated for SVT to be transported to a hospital; however, in some uncomplicated cases, the treat-and-release protocol could be instituted. The treat-and-release events were analysed and the conclusion was that only $13 \%$ of patients required EMS re-presentation and only 5.3\% required emergency department admission.

\section{CONCLUSIONS}

The knowledge of Polish paramedics and nurses concerning the emergency treatment of tachycardia is unsatisfactory in many aspects. Better undergraduate and postgraduate training on advanced life support guidelines should be recommended.

Conflict of interest: The authors declare no conflict of interest. The study was conducted without any commercial or financial relationships or financial support.

Acknowledgements: The authors would like to thank all the paramedics and nurses for their participation in the questionnaire survey.

\section{REFERENCES}

1. Page RL, Joglar JA, Caldwell MA, et al. Evidence Review Committee Chairf. 2015 ACC/AHA/HRS Guideline for the Management of Adult Patients With Supraventricular Tachycardia: A Report of the American College of Cardiology/American Heart Association Task Force on Clinical Practice Guidelines and the Heart Rhythm Society. Circulation. 2016; 133(14): e506-e574, doi: 10.1161/CIR.0000000000000311, indexed in Pubmed: 26399663.

2. Walfridsson U, Strömberg $A$, Janzon $M$, et al. Wolff-Parkinson-White syndrome and atrioventricular nodal re-entry tachycardia in a Swedish population: consequences on health-related quality of life. Pacing 
Clin Electrophysiol. 2009; 32(10): 1299-1306, doi: 10.1111/j.15408159.2009.02476.x, indexed in Pubmed: 19702600.

3. Luber $S$, Brady WJ, Joyce T, et al. Paroxysmal supraventricular tachycardia: outcome after ED care. Am J Emerg Med. 2001; 19(1): 40-42, doi: 10.1053/ajem.2001.20030, indexed in Pubmed: 11146016.

4. Roth A, Elkayam I, Shapira I, et al. Effectiveness of prehospital synchronous direct-current cardioversion for supraventricular tachyarrhythmias causing unstable hemodynamic states. Am J Cardiol. 2003; 91(4): 489-491, indexed in Pubmed: 12586276.

5. Neumar RW, Otto CW, Link MS, et al. Part 8: adult advanced cardiovascular life support: 2010 American Heart Association Guidelines for Cardiopulmonary Resuscitation and Emergency Cardiovascular Care. Circulation. 2010; 122(18 Suppl 3): S729-S767, doi: 10.1161/ CIRCULATIONAHA.110.970988, indexed in Pubmed: 20956224.

6. Soar J, Nolan JP, Böttiger BW, et al. Adult advanced life support section Collaborators. European Resuscitation Council Guidelines for Resuscitation 2015: Section 3. Adult advanced life support. Resuscitation. 2015; 95: 100-147, doi: 10.1016/j.resuscitation.2015.07.016, indexed in Pubmed: 26477701.

7. Honarbakhsh S, Baker V, Kirkby C, et al. Safety and efficacy of paramedic treatment of regular supraventricular tachycardia: a randomised controlled trial. Heart. 2017; 103(18): 1413-1418, doi: 10.1136/ heartjnl-2016-309968, indexed in Pubmed: 27613170.

8. Park J, Hwang SY, Shin TG, et al. Emergency medical service personnel need to improve knowledge and attitude regarding prehospital sepsis care. Clin Exp Emerg Med. 2017; 4(1): 48-55, doi: 10.15441/ ceem.16.159, indexed in Pubmed: 28435902.
9. Smith G, McD Taylor D, Morgans A, et al. Prehospital management of supraventricular tachycardia in Victoria, Australia: epidemiology and effectiveness of therapies. Emerg Med Australas. 2014; 26(4): 350-355, doi: 10.1111/1742-6723.12248, indexed in Pubmed: 24931380.

10. Clementy N, Fourquet $A$, Andre $C$, et al. Benefits of an early management of palpitations. Medicine (Baltimore). 2018; 97(28): e11466, doi: 10.1097/MD.0000000000011466, indexed in Pubmed: 29995805.

11. Goebel PJ, Daya MR, Gunnels MD. Accuracy of arrhythmia recognition in paramedic treatment of paroxysmal supraventricular tachycardia: a ten-year review. Prehosp Emerg Care. 2004; 8(2): 166-170, indexed in Pubmed: 15060851.

12. Smith G. Management of supraventricular tachycardia using the Valsalva manoeuvre: a historical review and summary of published evidence. Eur J Emerg Med. 2012; 19(6): 346-352, doi: 10.1097/ MEJ.0b013e32834ec7ad, indexed in Pubmed: 22186147.

13. Smith G, Boyle MJ. A cross-sectional study of Victorian Mobile Intensive Care Ambulance Paramedics knowledge of the Valsalva Manoeuvre. BMC Emerg Med. 2009; 9: 23, doi: 10.1186/1471-227X-9-23, indexed in Pubmed: 20003461.

14. Carlson JN, Karns C, Mann NC, et al. Procedures Performed by Emergency Medical Services in the United States. Prehosp Emerg Care. 2016; 20(1): 15-21, doi: 10.3109/10903127.2015.1051682, indexed in Pubmed: 26270634.

15. Minhas $R$, Vogelaar $G$, Wang $D$, et al. A prehospital treat-and-release protocol for supraventricular tachycardia. CJEM. 2015; 17(4): 395-402, doi: 10.1017/cem.2014.53, indexed in Pubmed: 26134056. 